\title{
Parameter Estimation Based on Linear Regression for Commercial Quadrotors *
}

\author{
João R. S. Benevides* Roberto S. Inoue ** \\ Marlon A. D. Paiva ${ }^{* * *}$ Marco H. Terra ${ }^{* * * *}$ \\ * Departamento de Engenharia Elétrica e de Computação, \\ Universidade de São Paulo, SP, (e-mail: jrsbenevides@usp.br). \\ ** Departamento de Engenharia Elétrica, Universidade Federal de São \\ Carlos, SP, (e-mail: rsinoue@ufscar.br). \\ *** Departamento de Engenharia Elétrica e de Computação, \\ Universidade de São Paulo, SP, (e-mail: dalsochio@usp.br). \\ **** Departamento de Engenharia Elétrica e de Computação, \\ Universidade de São Paulo,SP, (e-mail: terra@sc.usp.br).
}

\begin{abstract}
Over the last decade, the rapid evolution in communications technology, mechanics and electronics has propelled access to Unmanned Aerial Vehicles (UAVs). Among this class of robots, quadrotors stand out due to maneuverability and Vertical Take Off and Landing (VTOL) capability. This work proposes a methodology to solve the model parameter estimation problem of a commercial quadrotor. Mathematical models are derived and implementation details are given. Experimental results, obtained in an open source environment associated with a motion capture system, verify that the estimated dynamic model approximates to the real dynamics.
\end{abstract}

Keywords: UAV, Quadrotor, Parameter estimation

\section{INTRODUCTION}

Over the last two decades, Unmanned Aerial Vehicles (UAVs) have drawn attention to both military and civil research applications. Recent surveys reveal that the wide use of drones in public safety and precision agriculture has an yearly economic impact of billions of dollars in the U.S. and Britain alone.

When compared to fixed-wing aircrafts, quadrotors stand out due to their simple mechanical design, maneuverability, Vertical Take Off and Landing (VTOL) capability and reduced cost. They normally come equipped with on-board sensory and communicate wirelessly with a central station. Four rotating blades enable flight in a way similar to that of a helicopter as movement is attained by changing the speeds of each rotor, creating different thrust forces along the three axes. As described in the work of Hernandez et al. (2013), the quadrotor can fly both indoor and outdoor and is able to perform aggressive aerial maneuvers as well as keep stable, hovering and carry out precise flight paths.

However, the complex, non-linear and underactuated dynamic showcased by such aerial vehicles complicate the tasks of modelling and identification. Nonetheless, many researchers propose techniques that overcome such difficulties and provide methodologies for estimating unknown model parameters.

In recent years, commercial quadrotors have become more affordable with low-cost versions that feature a high attitude stability. Whilst model-based control systems are

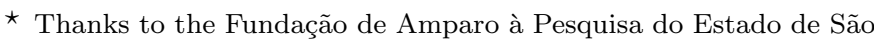
Paulo - FAPESP, for the grant under project n. 2017/05668-0
}

generally associated to control performance, these might lead to a heavy computational burden, as pointed out by Santoso et al. (2015). In this sense, some works are devoted to simplifying the complex model of UAVs. Morar and Nascu (2012) derive a simplified non-linear model kinematic and dynamic representation of a Parrot ARDrone. Kim et al. (2009) consider only $z$-directional linear motions and, by streamlining the non-linear dynamic to a simple relation of acceleration, velocity and command inputs, Santana et al. (2014) design a control strategy, also for a Parrot AR-Drone.

With this motivation, this work stands out by providing a formulation for computing unknown parameters in this simplified dynamic model. Real flight data is acquired and details regarding experimental implementation provide directions for a proper estimation of the parameters. Based on the work developed in Benevides et al. (2019), where a ROS-based package for controller strategies evaluation and autonomous flight is implemented, experimental results are obtained. Real data is compared to the one provided by the estimated model and results are then discussed.

For simplification of notation, $\cos (\psi)$ and $\sin (\psi)$ are denoted by $c(\psi)$ and $s(\psi)$, respectively.

\section{BEBOP 2 QUADROTOR PLATFORM}

The Parrot Bebop 2 is an affordable ${ }^{1}$ leisure quadcopter equipped with a high-resolution camera. It offers a highlevel flight stability, embedded IMU and GPS and also allows piloting with a smartphone or tablet through $\mathrm{Wi}$ Fi connection.

$1 \mathrm{U} \$ 349,99$ as in Parrot's official website 


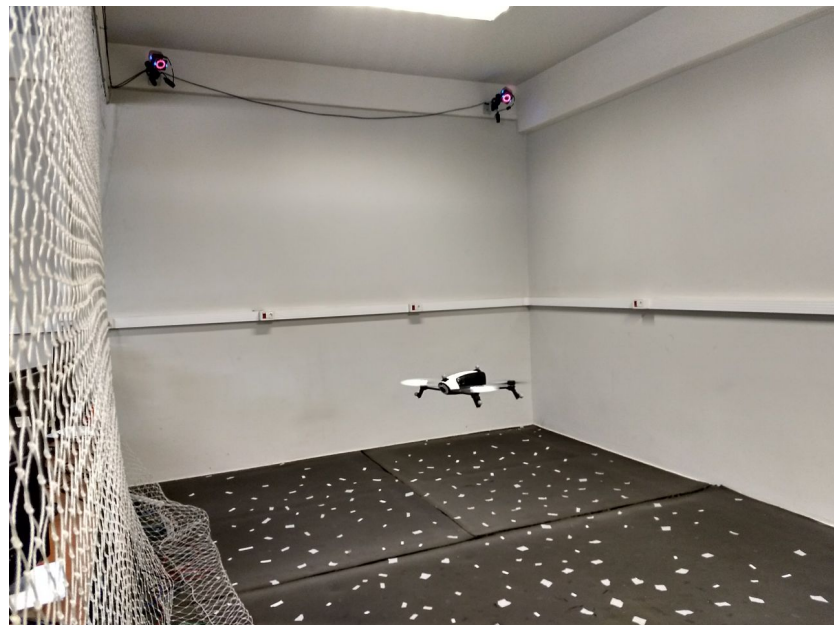

Figure 1. Indoor scenario with a Parrot Bebop 2 in a Vicon equiped room.

A driver for the Robot Operating System (ROS), based on Parrot's official Software Development Kit, has been developed in Benevides et al. (2019) and made available as open source under the name drone_dev. This brings a key advantage of dealing with the Parrot Bebop 2 for research purposes, as minor time is spent in setting a suitable ROS compatible flight environment. In fact, the ROS package, among other parameters, is responsible for delivering pose estimation and sending input commands.

Among the features of the drone_dev, we highlight the capability of evaluating controller strategies and performing trajectory tracking tasks both in outdoor and indoor scenarios. It is worth mentioning that a Vicon motion capture system is responsible for estimating position and orientation in tests performed in indoor environments.

\section{MATHEMATICAL MODEL OF A COMMERCIAL QUADROTOR}

Let $\{W\}$ and $\{B\}$ to be the global coordinate frame and the the local (body) coordinate frame, respectively. Consider now a given point $P$ in space, described both in local and global frames as ${ }^{B} P$ and ${ }^{W} P$, respectively. The translation vector ${ }^{W} P_{B O R G}$ locates the origin of $\{B\}$ relative to $\{W\}$ as depicted in Figure 2 .

Denoting ${ }_{B}^{W} R$ as the rotation matrix that describes $\{B\}$ relative to $\{W\}$, then

$$
{ }^{W} P={ }_{B}^{W} R{ }^{B} P+{ }^{W} P_{B O R G}
$$

Computing the first and second derivatives of (1) yields in

$$
\begin{aligned}
W \ddot{P} & ={ }_{B}^{W} R^{B} \dot{P} \\
& \ddot{P}={ }_{B}^{W} R^{B} \ddot{P}
\end{aligned}
$$

Let us now define augmented states $q_{g}=\left[\begin{array}{llll}x_{g} & y_{g} & z_{g} & \psi_{g}\end{array}\right]^{T}$ and $q_{b}=\left[\begin{array}{llll}x_{b} & y_{b} & z_{b} & \psi_{b}\end{array}\right]^{T}$ to represent global and local pose, respectively. The yaw angles are defined by $\psi_{g}$ and $\psi_{b}$ in global and local terms, respectively. For our case, where attitude stability is assumed guaranteed by embedded control, we only consider rotation in $z$ axis. Accordingly,

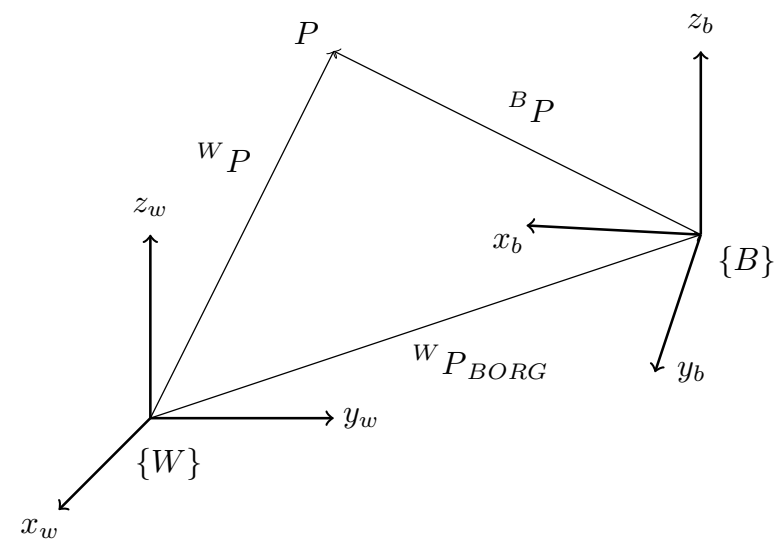

Figure 2. The global frame is denoted as $\{W\}$ while $\{B\}$ stands for the body frame.

yaw angles are assumed to be identical, i.e. $\psi_{g}=\psi_{b}$. Then, by defining an augmented rotation matrix $R_{t}$ as

$$
R_{t}=\left[\begin{array}{cc}
{ }_{B} R & 0 \\
\mathbf{0} & 1
\end{array}\right],
$$

we can finally obtain the relations

$$
\begin{aligned}
& \dot{q}_{g}=R_{t} \dot{q}_{b} \\
& \ddot{q}_{g}=R_{t} \ddot{q}_{b}
\end{aligned}
$$

where

$$
R_{t}=\left[\begin{array}{cccc}
c(\psi) & -s(\psi) & 0 & 0 \\
s(\psi) & c(\psi) & 0 & 0 \\
0 & 0 & 1 & 0 \\
0 & 0 & 0 & 1
\end{array}\right]
$$

Despite the inherent complex dynamics of quadrotors, Santana et al. (2014) and references therein adopt a simplified dynamic equation for a Parrot AR.Drone and general quadrotors. This formulation is a model that enables position control with velocity input vector $u=$ $\left[\begin{array}{llll}u_{v_{x}} & u_{v_{y}} & u_{v_{z}} & u_{v_{\psi}}\end{array}\right]^{T}$ and is described by

$$
\begin{aligned}
& \ddot{x}_{b}=\gamma_{1} u_{v_{x}}-\gamma_{2} \dot{x}_{b} \\
& \ddot{y}_{b}=\gamma_{3} u_{v_{y}}-\gamma_{4} \dot{y}_{b} \\
& \ddot{z}_{b}=\gamma_{5} u_{v_{z}}-\gamma_{6} \dot{z}_{b} \\
& \ddot{\psi}_{b}=\gamma_{7} u_{v_{\psi}}-\gamma_{8} \dot{\psi}_{b}
\end{aligned},
$$

where $\gamma_{i}$, with $i=\{1,2, \ldots, 8\}$, are coefficients to be identified. This can be rewritten in matrix form as

$$
\ddot{q}_{b}=\Gamma_{A} \dot{q}_{b}+\Gamma_{B} u
$$

where

$$
\Gamma_{A}=\left[\begin{array}{cccc}
-\gamma_{2} & 0 & 0 & 0 \\
0 & -\gamma_{4} & 0 & 0 \\
0 & 0 & -\gamma_{6} & 0 \\
0 & 0 & 0 & -\gamma_{8}
\end{array}\right] \text { and } \Gamma_{B}=\left[\begin{array}{cccc}
\gamma_{1} & 0 & 0 & 0 \\
0 & \gamma_{3} & 0 & 0 \\
0 & 0 & \gamma_{5} & 0 \\
0 & 0 & 0 & \gamma_{7}
\end{array}\right]
$$


Using the relations derived in (3) and substituting into (4) yields in

$$
\ddot{q}_{g}=R_{t} \Gamma_{A} R_{t}^{T} \dot{q}_{g}+R_{t} \Gamma_{B} u
$$

Notice that the form of (5) is useful as the orthogonality properties of $R_{t}$ can be seized in order to simplify computations. In fact, (5) is a similarity transformation of (4) that can be rewritten as

$$
\begin{aligned}
& \ddot{x}_{g}=\gamma_{1} u_{v_{x}} c\left(\psi_{b}\right)-\gamma_{2} \dot{x}_{b} c\left(\psi_{b}\right)-\gamma_{3} u_{v_{y}} s\left(\psi_{b}\right)+\gamma_{4} \dot{y}_{b} s\left(\psi_{b}\right) \\
& \ddot{y}_{g}=\gamma_{1} u_{v_{x}} s\left(\psi_{b}\right)-\gamma_{2} \dot{x}_{b} s\left(\psi_{b}\right)+\gamma_{3} u_{v_{y}} c\left(\psi_{b}\right)-\gamma_{4} \dot{y}_{b} c\left(\psi_{b}\right) \\
& \ddot{z}_{g}=\gamma_{5} u_{v_{z}}-\gamma_{6} \dot{z}_{b} \\
& \ddot{\psi}_{g}=\gamma_{7} u_{v_{\psi}}-\gamma_{8} \dot{\psi}_{b}
\end{aligned}
$$

\section{FLIGHT DATA ACQUISITION}

Experimental results were collected in an indoor environment, where the quadcopter pose was estimated by a Vicon motion tracking system comprised of four cameras. A host $\mathrm{PC}$ running the drone_dev developed in Benevides et al. (2019), a ROS package for evaluating controller strategies, was used to perform trajectory tracking of a reference and to estabilish communication with the quadrotor and Vicon system. The setup of the flight tests are illustrated according to Fig. 3 .

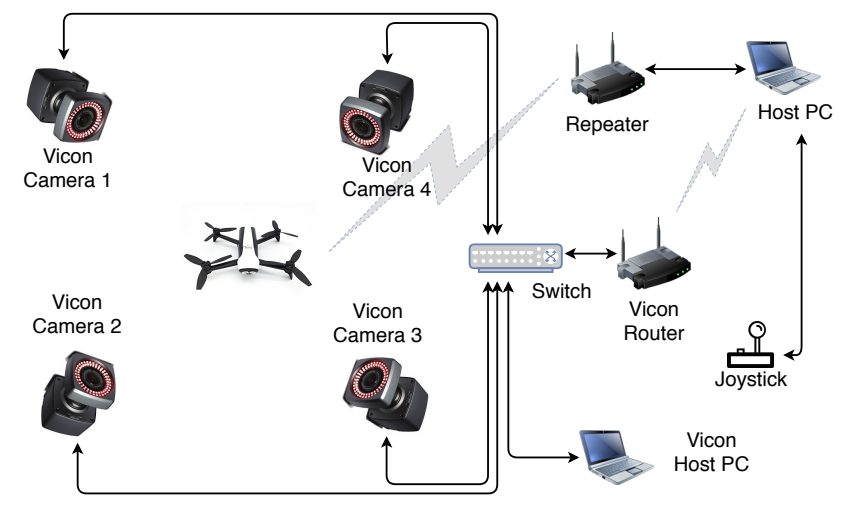

Figure 3. A diagram representing the setup of an indoor flight.

Recalling the dynamic model in (6), we can divide the estimation task into three independent steps. A first flight is responsible for sending inputs in $x$ and $y$ directions. Because parameters $\left\{\gamma_{1}, \gamma_{2}, \gamma_{3}, \gamma_{4}\right\}$ are coupled between those two axes, sending signals simultaneously in both channels yields in better estimation results. A second flight excites $z$ axis and a last flight moves around $\psi$ direction. It is known from parameter identification theory that the chosen input signal should have enough frequencies in order to excite all the modes in the system, as highlighted in the work of Carro Pérez et al. (2014). Therefore, we have chosen a sinusoidal signal with two non-multiple frequencies as

$$
\begin{aligned}
u_{v_{x}} & =0.1[s(1.3 t)+s(t)] \\
u_{v_{y}} & =0.1[c(1.3 t)+c(t)] \\
u_{v_{z}} & =0.1[s(1.3 t)+s(t)] \\
u_{v_{\psi}} & =0.6[s(1.3 t)+s(t)]
\end{aligned}
$$

\section{PARAMETER ESTIMATION}

Here we propose the utilization of linear regression using least squares method in order to estimate $\gamma$ parameters in (6). By sending basic input velocity commands, namely, input $\nu$, and logging correspondent position and attitude data. After computing a collection of input-output relations, we estimate the parameters by computing the solution to the problem.

Let $n_{x}, n_{y}, n_{z}$ and $n_{\psi}$ be the number of samples collected for training for each of the elements of $\ddot{q}_{g}$ in $(6)$. In order to help with the setting of regression matrices, we can define $\alpha^{i}$ coefficients and compute them, at each iteration $i$, as shown below.

$$
\begin{array}{lll}
\alpha_{1}^{i}:=u_{v_{x}}^{i} c\left(\psi^{i}\right), & \alpha_{5}^{i}:=u_{v_{x}}^{i} s\left(\psi^{i}\right), & \alpha_{9}^{i}:=u_{\dot{z}}^{i}, \\
\alpha_{2}^{i}:=-v_{x}^{i} c\left(\psi^{i}\right), & \alpha_{6}^{i}:=-v_{x}^{i} s\left(\psi^{i}\right), & \alpha_{10}^{i}:=-\dot{z}^{i}, \\
\alpha_{3}^{i}:=-u_{v_{y}}^{i} s\left(\psi^{i}\right), & \alpha_{7}^{i}:=u_{v_{y}}^{i} c\left(\psi^{i}\right), & \alpha_{11}^{i}:=u_{\dot{\psi}}^{i}, \\
\alpha_{4}^{i}:=v_{y}^{i} s\left(\psi^{i}\right), & \alpha_{8}^{i}:=-v_{y}^{i} c\left(\psi^{i}\right), & \alpha_{12}^{i}:=-\dot{\psi}^{i} .
\end{array}
$$

Therefore, one can rewrite (6) and generically formulate regression matrices as

$$
\begin{gathered}
{\left[\begin{array}{c}
\ddot{x}_{g}^{1} \\
\vdots \\
\ddot{x}_{g}^{n_{x}} \\
\ddot{y}_{g}^{1} \\
\vdots \\
\ddot{y}_{g}^{n_{y}}
\end{array}\right]=\left[\begin{array}{ccccc}
1 & \alpha_{1}^{1} & \alpha_{2}^{1} & \alpha_{3}^{1} & \alpha_{4}^{1} \\
1 & \alpha_{1}^{2} & \alpha_{2}^{2} & \alpha_{3}^{2} & \alpha_{4}^{2} \\
\vdots & \vdots & \vdots & \vdots & \vdots \\
1 & \alpha_{1}^{n_{x}} & \alpha_{2}^{n_{x}} & \alpha_{3}^{n_{x}} & \alpha_{4}^{n_{x}} \\
1 & \alpha_{5}^{1} & \alpha_{6}^{1} & \alpha_{7}^{1} & \alpha_{8}^{1} \\
1 & \alpha_{5}^{2} & \alpha_{6}^{2} & \alpha_{7}^{2} & \alpha_{8}^{2} \\
\vdots & \vdots & \vdots & \vdots & \vdots \\
1 & \alpha_{5}^{n_{y}} & \alpha_{6}^{n_{y}} & \alpha_{7}^{n_{y}} & \alpha_{8}^{n_{y}}
\end{array}\right]\left[\begin{array}{c}
0 \\
\gamma_{1} \\
\gamma_{2} \\
\gamma_{3} \\
\gamma_{4}
\end{array}\right],} \\
{\left[\begin{array}{c}
\ddot{z}_{g}^{1} \\
\vdots \\
\ddot{z}_{g}^{n_{z}}
\end{array}\right]=\left[\begin{array}{cccc}
1 & \alpha_{9}^{1} & \alpha_{10}^{1} \\
1 & \alpha_{9}^{2} & \alpha_{10}^{2} \\
\vdots & \vdots & \vdots \\
1 & \alpha_{9}^{n_{z}} & \alpha_{10}^{n_{z}}
\end{array}\right]\left[\begin{array}{c}
0 \\
\gamma_{5} \\
\gamma_{6}
\end{array}\right]}
\end{gathered}
$$

and

$$
\left[\begin{array}{c}
\ddot{\psi}_{g}^{1} \\
\vdots \\
\ddot{\psi}_{g}^{n_{\psi}}
\end{array}\right]=\left[\begin{array}{ccc}
1 & \alpha_{11}^{1} & \alpha_{12}^{1} \\
1 & \alpha_{11}^{2} & \alpha_{12}^{2} \\
\vdots & \vdots & \vdots \\
1 & \alpha_{11}^{n_{\psi}} & \alpha_{12}^{n_{\psi}}
\end{array}\right]\left[\begin{array}{c}
0 \\
\gamma_{7} \\
\gamma_{8}
\end{array}\right]
$$

Because (8), (9) and (10) are in the form $y=X \hat{\theta}$, one can estimate $\gamma$ parameters by computing $\hat{\theta}=\left(X^{T} X\right)^{-1} X^{T} y$ in each equation. This is the known solution to the ordinary least squares problem, which minimizes the sum of square residuals. Solving for the data experimentally acquired on flight, we finally obtained

$$
\gamma=\left[\begin{array}{llllllll}
4.33 & 0.23 & 4.12 & 0.41 & 4.42 & 3.13 & 5.92 & -0.38
\end{array}\right]
$$


It is important to point out that input and output data are logged at different rates, which is handled, in our case, by comparing time stamps. In fact, the parameter estimation processing is entirely done offline as information of velocity and acceleration are needed. These are obtained by differentiating position and orientation signals through polynomial approximation.

\section{RESULTS}

After estimating $\gamma$ parameters, it is necessary to validate them by comparing with real flight data with a model, calculated with the parameters obtained. For that purpose, we again performed three different flights in order to highlight the effectiveness of the estimation.

First, a 200s-duration flight was performed in the $x y$ plane. A long observation period is justified due to the simplified dynamic model neglects the influence of pitch and roll angles.

Two other flights were performed in order to analyze behavior in $z$ and $\psi$ axes, each of them during $100 \mathrm{~s}$. The trajectories chosen are not well behaved in order to excite non-linearities. Notice that for some time intervals, estimation differs completely from real data. Results are summarized in Figure 4.

We can notice that some low and large spikes exist along the estimated curve in every axis. This is due to the fact that only position feedback is provided by the Vicon motion tracking system. A workaround is the design of a Kalman Filter for velocity and acceleration estimation, which is done when implementing control strategies with the drone_dev package.

Although the proposed derivations and methodology for estimating unknown parameters cannot replace a complete non-linear model, the results obtained when comparing the accelerations in both axes, however, suggest the computed parameters provide a close approximation to the real dynamics. Furthermore, this formulation and results are extended to commercial quadrotors with attitude flight stability and similar input-output relation as the one presented in this work.

\section{CONCLUSION}

This work proposed a method for estimating parameters of a commercial quadrotor in indoor scenarios. In order to achieve this goal, the simplified dynamic model was rewritten in the form of regression matrices which admit a single solution for a data collection. Moreover, we provided rich details regarding the nature of the chosen inputs, organization of flight tests and validation of the proposed model. For that purpose, an experimental scenario was set and an open source ROS-based package was used for obtaining the input-output relation. Although a Parrot Bebop 2 quadcopter was used in the experiments, the formulations and considerations derived here extend to other off-the-shelf quadrotors driven by similar input commands and stable hovering flight. The results obtained reveal that the simple dynamic model combined with the proposed parameter estimation provide a close approximation of the complex real dynamics of such vehicles.
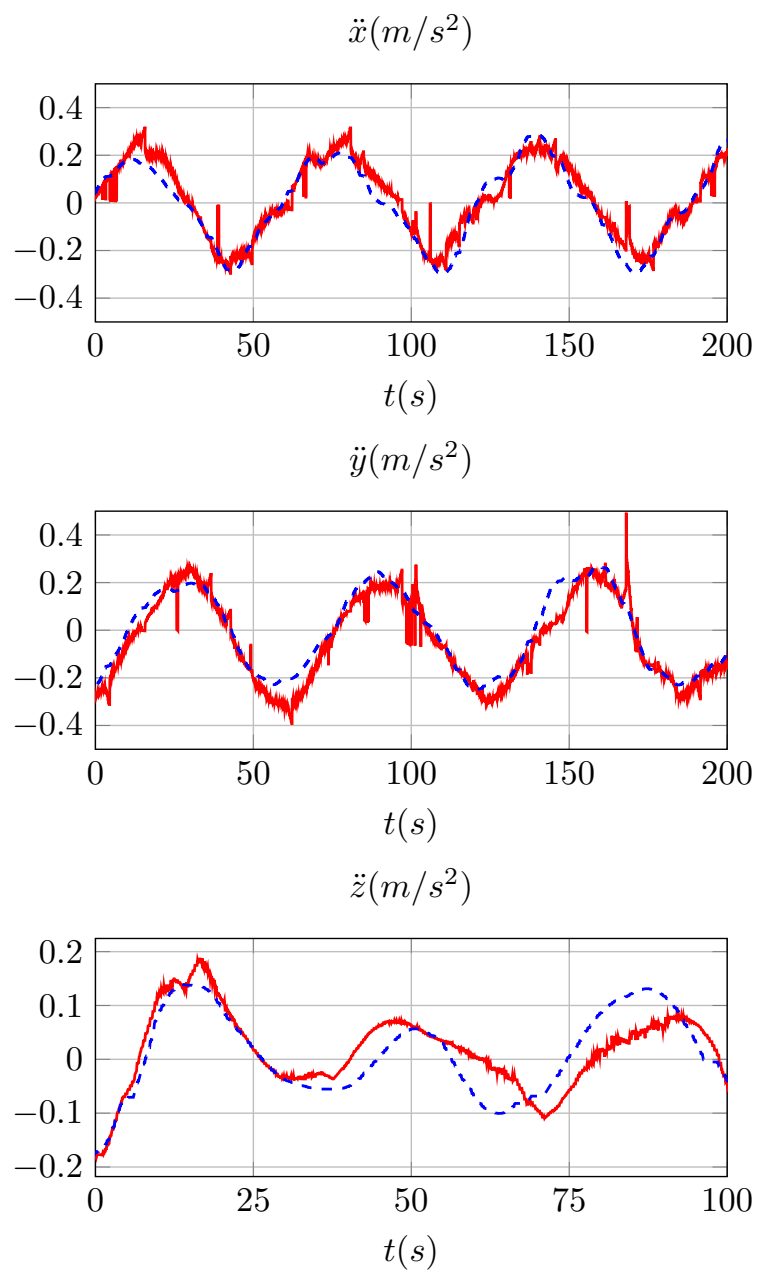

$\ddot{\psi}\left(\mathrm{rad} / \mathrm{s}^{2}\right)$

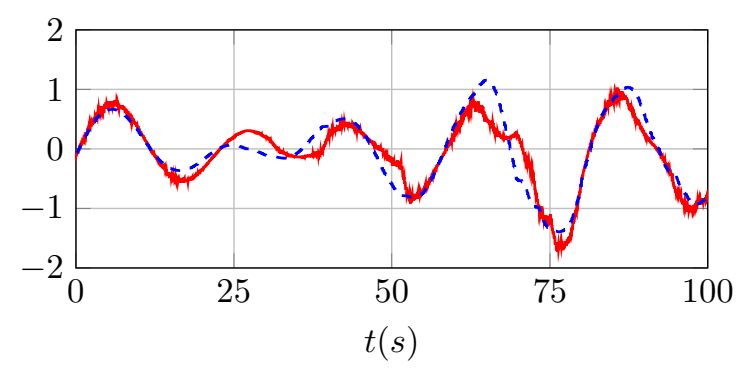

Estimated - - - Real

Figure 4. Results compare real data and estimation computed by parameters obtained with previous flights.

In order to extend this work, we are considering more complex mathematical models in order to improve the dynamic model and overall performance, especially in $z$ and $\psi$ axes. Finally, we are currently investigating other methods for online parameter estimation under wind disturbances and their implementation on the Parrot Bebop 2 and other commercial quadrotors.

\section{ACKNOWLEDGMENT}

We thank the two anonymous reviewers whose recommendations have greatly improved the quality of this work. 


\section{REFERENCES}

Benevides, J.R.S., Inoue, R.S., Paiva, M.A.D., and Terra, M.H. (2019). ROS-Based Robust and Recursive Optimal Control of Commercial Quadrotors. In Proc. of the 15th International Conference on Automation Science and Engineering (CASE) - Accepted for publishing.

Carro Pérez, I., Flores-Araiza, D., Fortoul-Díaz, J.A., Maximo, R., and Gonzalez-Hernandez, H.G. (2014). Identification and pid control for a quadrocopter. In 2014 International Conference on Electronics, Communications and Computers (CONIELECOMP), 77-82. doi:10.1109/CONIELECOMP.2014.6808571.

Hernandez, A., Copot, C., De Keyser, R., Vlas, T., and Nascu, I. (2013). Identification and path following control of an ar.drone quadrotor. In 2013 17th International Conference on System Theory, Control and Computing (ICSTCC), 583-588. doi:10.1109/ICSTCC. 2013.6689022.

Kim, J., Kang, M.S., and Park, S. (2009). Accurate modeling and robust hovering control for a quad-rotor vtol aircraft. Journal of Intelligent and Robotic Systems, 57(1), 9. doi:10.1007/s10846-009-9369-z.

Morar, I. and Nascu, I. (2012). Model simplification of an unmanned aerial vehicle. In Proceedings of 2012 IEEE International Conference on Automation, Quality and Testing, Robotics, 591-596. doi:10.1109/AQTR.2012. 6237779

Santana, L.V., Brandao, A.S., Sarcinelli-Filho, M., and Carelli, R. (2014). A trajectory tracking and 3D positioning controller for the AR.Drone quadrotor. 2014 International Conference on Unmanned Aircraft Systems, ICUAS 2014 - Conference Proceedings, 756-767. doi: 10.1109/ICUAS.2014.6842321.

Santoso, F., Garratt, M.A., and Anavatti, S.G. (2015). Fuzzy logic-based self-tuning autopilots for trajectory tracking of a low-cost quadcopter: A comparative study. In 2015 International Conference on Advanced Mechatronics, Intelligent Manufacture, and Industrial Automation (ICAMIMIA), 64-69. doi:10.1109/ ICAMIMIA.2015.7508004. 